\title{
Similarity and Difference in Time Structures of Gamma-Ray Flares in the Crab Nebula at Drastically Differing Energies
}

\author{
A.S. Lidvansky \\ Institute for Nuclear Research, \\ Russian Academy of Sciencess, Moscow, Russia \\ E-mail: lidvanskel ebedev.ru
}

\begin{abstract}
Characteristics of the flares of gamma rays detected from the Crab Nebula by the AGILE and Fermi-LAT satellite instruments are compared with those of a gamma ray burst recorded by several air shower arrays on February 23, 1989. It is demonstrated that though pulsar-periodicity and energy spectra of emissions at $100 \mathrm{MeV}$ (satellite gamma ray telescopes) and $100 \mathrm{TeV}$ (EAS arrays) are different, their time structures seem to be similar. Moreover, may be the difference between "flares" and "waves" recently found in the Crab Nebula emission by the AGILE team also exists at ultra-high energies.
\end{abstract}




\section{Introduction}

Gamma-ray flares from the Crab Nebula were discovered in a few-hundred-MeV energy range by the AGILE [1] and Fermi LAT [2] satellite telescopes. Since then, both the telescopes continue recording such flares approximately once in a year, the strongest of them (super-flare) having been detected in April 2011 [3], [4]. The energy spectra of additional emission during the flares were measured to be different from those of the Nebula, however, "the mechanism driving the flares, their impulsive nature, the 12-month recurrence time, and the location, remain unknown" [5]. For all that, based on multi-wavelength campaign to study the Crab using Keck, Hubble Space Telescope (HST), and Chandra X-ray Observatory, the authors of [5] suggested the so-called "inner knot" to be just the emitting region for the flares. In addition, the analysis made by the AGILE collaboration for the September-October 2007 event has found a fine structure in the flare's time behavior [6]. They have demonstrated that there is a difference between shorter "flares" and longer "waves" in the Crab Nebula emission during this flare.

After discovery of gamma ray flares by AGILE and Fermi LAT it was recalled [7] that one event of this type (though at much higher energies) had been discovered many years ago by several EAS arrays [8], [9], [10].

\section{Gamma-Ray Emission Burst on February 23, 1989}

First announcement about this burst was made during the International Workshop on Gamma-Ray Astronomy in Crimea in 1989 [8]. An increase of intensity of extensive air showers (EAS) was detected by the Carpet air shower array of the Baksan Neutrino Observatory on February 23, 1989. After this communication the group at Kolar Gold Fields (KGF) in India confirmed this result of Baksan and published a paper [9] on simultaneous detection of a gamma-ray burst in the Crab Nebula at ultra high-energies independently by two EAS arrays. Final publication [10] by the Baksan and Durham University teams summarized the data of all arrays that could observe the source on this day. It was demonstrated that with different significance the burst was detected by all air shower arrays located in the longitude range from India to Italy (KGF, Tien Shan, Baksan and EAS TOP). The arrays located to the east and west from this interval (at that time OHYA MINE and Akeno SPICA in Japan and HEGRA at La Palma, Canary Islands, were in operation) showed no excess from the source direction. Thus, the total duration of the observed effect was no longer than about 7 hours. One can speak also about a possible decay of intensity whose maximum probably fell on observations with the KGF array. Figures 1, 2, and 3 taken from paper [10] demonstrate the most remarkable features of the burst as detected by the Baksan Carpet air shower array. It is clearly seen that the Baksan array data demonstrate obvious dependence of the effect on the pulsar phase: events from a single phase bin can produce the entire observed excess of intensity (the KGF group also found some phase irregularity, though not so well pronounced).

Summarizing, one can state that with a rather high probability (combined probability of random coincidences was estimated in [10] as $1.25 \times 10^{-7}$ ) a gamma-ray burst from the Crab Nebula was detected on February 23, 1989 in the energy range $10^{14}-10^{15} \mathrm{eV}$, and excess emission in this burst is somehow connected with the pulsar's activity. 

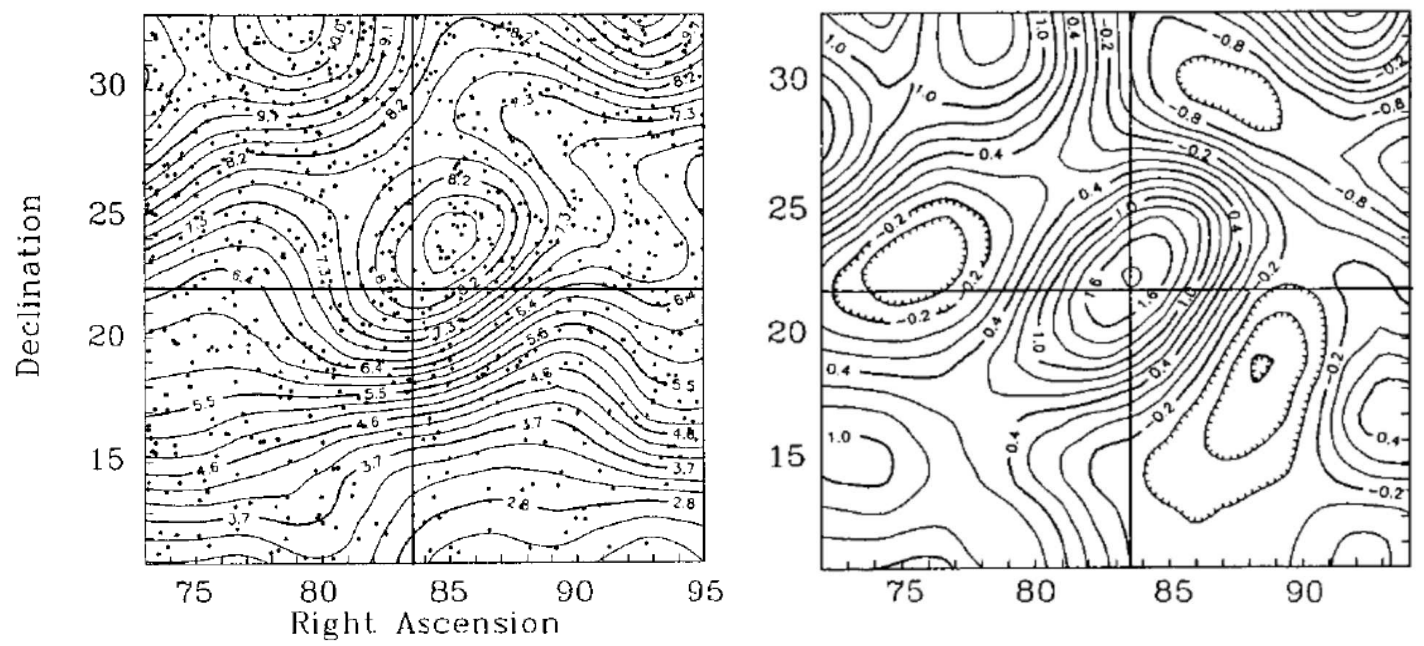

Figure 1: Left panel: Arrival directions of showers in a $22^{\circ} \times 22^{\circ}$ area in equatorial coordinates about the Crab direction (central cross). Individual events as well as smoothed density contours are presented. Labels give the density of showers per four square degrees. Right panel: The same area as in Fig. 2, but only excess density of showers above background is shown. Maximum of density now is even better coincides with Crab coordinates.

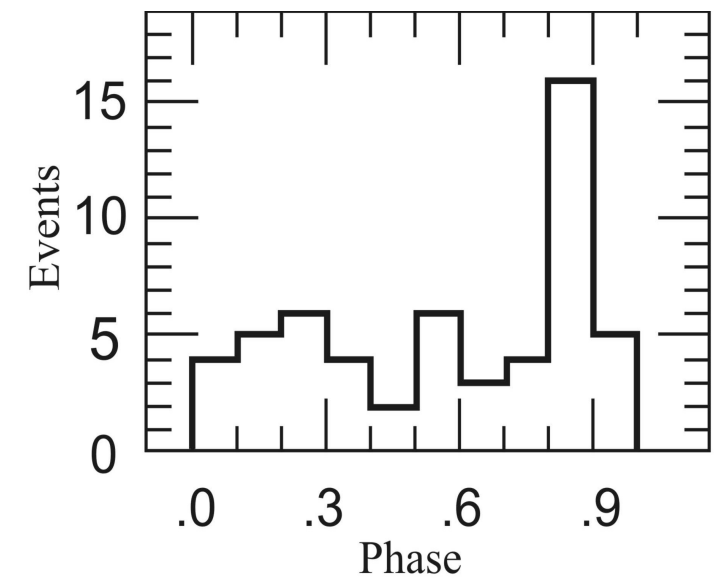

Figure 2: The relative phase distribution of the 55 events within the Crab cell after barycentring the arrival times and applying the values of the pulsar period and its derivative according to the Jodrell Bank Crab ephemeris.

\section{Different or Similar Types of Flares at Different Energies?}

In [7] attention was attracted to the fact that three-pulse temporal structure in the event of February 23, 1989 might be reproduced at least in one of the AGILE flare events (September 2007). A bit later it has been discovered [6] that in addition to flares there exists another type of intensity increase called waves. And exactly during the September 2007 three short flares (F1, F2, and F3) were identified together with two waves W1 and W2 (see Fig. 4).

Similarity of the temporal structures for events of Fig. 3 and Fig. 4 is obvious. In one case three bunches shorter than one hour are repeated with a period slightly longer than one hour. The other event includes three flares with duration of about one day, and they are repeated with a period a bit longer than one day. So, a sort of a stable pattern with a scale factor is observed at drastically different energies of gamma rays. It should be noted that the fine temporal structure was also reported by the Fermi LAT team for the March 2013 flare: "The light curve shows 
three sharp spikes (MJD 56357.1, 56357.9, 56360.1) on top of a strongly increased flux level." [11]. Here, the period between the spikes is not so constant, but its duration, nevertheless, is close to that of Fig. 4 event.

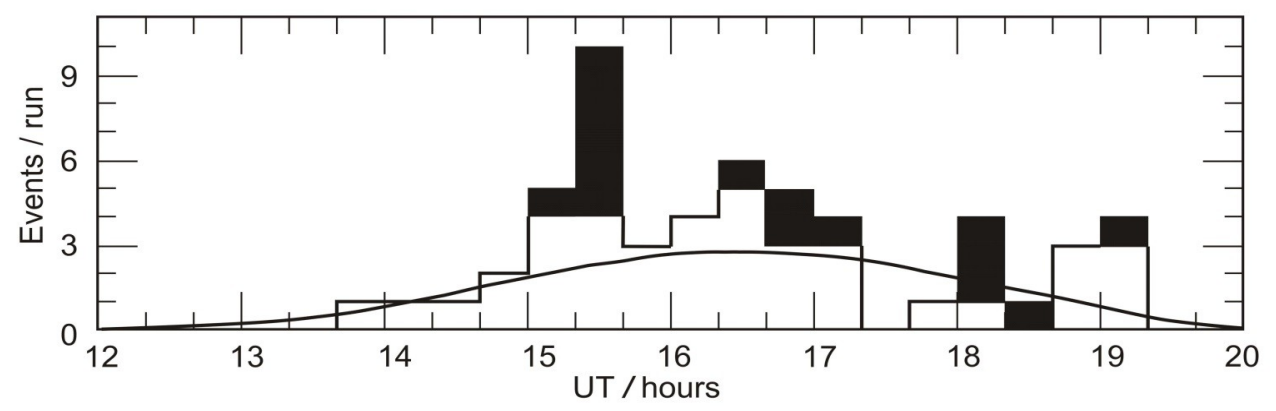

Figure 3: The number of events within the Crab cell per 20 min run for 23 February 1989. Smooth curve represents expected background. Blacked events are from the 9th bin in Fig. 2.

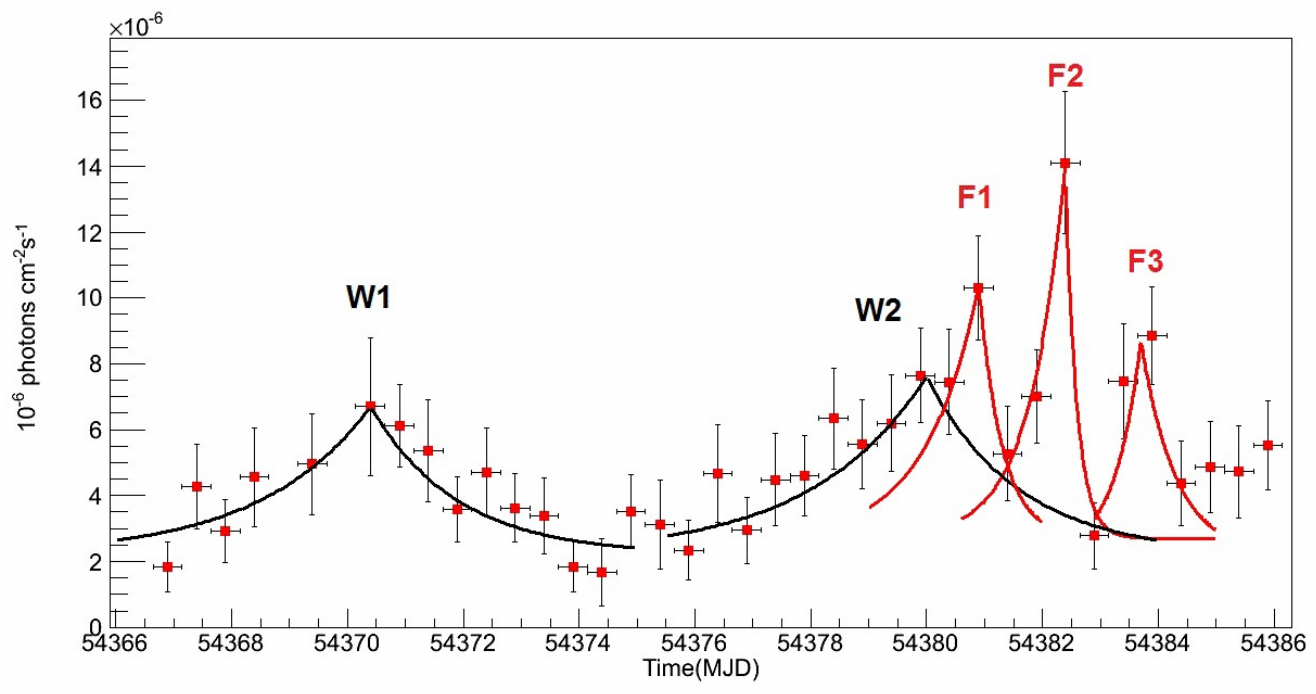

Figure 4: The difference between flares and waves in the Crab Nebula gamma-ray emission according to Striani et al. [6] (the September 2007 event). Three-flare structure of the event is similar to three bunches of narrow-phase emission constituting almost all excess counts in Fig. 3.

The Crab pulsar emission has very complicated energy dependence. In the radio waveband in addition to the main pulse (MP) and interpulse (IP) there are a precursor of the MP, lowfrequency component (LFC) and two high-frequency components (HFC1 and HFC2), as well as giant radio pulses that randomly appear on different phases. At higher energies the Crab light curve becomes more regular and the gamma-ray domain has only two pulses P1 and P2. The amplitudes of these two pulses depend on energy. For example, the P2 pulse is twice larger in amplitude than P1 at energy less than $10 \mathrm{MeV}$ (COMPTEL), but already at energy $>30 \mathrm{MeV}$ (EGRET) the situation is opposite. At the energy exceeding $100 \mathrm{GeV}$ (the data of Cherenkov telescopes VERITAS and MAGIC) pulse P2 becomes dominant again. There are also some 
indications to the possible appearance of a new pulse P3. So, it would be not surprising if at still higher energy only a single pulse survives, as it takes place in Fig. 2.

One can suggest that the source of high-energy gammas is really connected with the pulsar and acceleration of electrons near it. Then propagation to more distant areas of the Nebula where magnetic irregularities serve as targets for generation of lower energy gammas (it is generally believed that synchrotron mechanism is underlying for the flares) results in spreading of the signal with simultaneous randomization of the phase. In this way, probably, one could explain the main differences between flares at different energies, which are as follows: 1) at ultra-high energies the emission retains pulsar periodicity, which is not the case for gamma rays of a few-hundred-MeV energy; 2) the similar time structures of the Crab light curves have different time scales.

\section{Acknowledgments}

The work is partially supported by the Russian Foundation for Basic Research, grant no. 1602-00687.

\section{References}

[1] M. Tavani et al., Discovery of Powerful Gamma-Ray Flares from the Crab Nebula, Science 2011; 331: Issue 6018, 736-39.

[2] A.A. Abdo et al., Gamma-Ray Flares from the Crab Nebula, Science 2011; 331: 739-42.

[3] R. Buehler et al., Gamma-ray Activity in the Crab Nebula: The Exceptional Flare of 2011 April, Astrophys. Journ. 2012; 749: 26.

[4] E. Striani et al., The Crab Nebula Super-Flare in 2011 April: Extremely Fast Particle Acceleration and Gamma-Ray Emission, Astrophys. Journ. Lett. 2011; 741: L5.

[5] A. Rudy et al., Characterization of the Inner Knot of the Crab: The Site of the Gamma-ray Flares? Astrophys. Journ. 2015; 811: 24.

[6] E. Striani et al., Variable gamma-ray emission from the Crab Nebula: Short flares and long waves, Astrophys. Journ. 2013; 765: 52.

[7] A.S. Lidvansky, On the burst activity of the Crab Nebula and pulsar at high and ultra-high energies, Journal of Physics: Conference Series 2013; 409: 012114.

[8] V.V. Alexeenko, A.S. Lidvansky and V.A. Tizengauzen, $A$ Search for $>10^{14} \mathrm{eV}$ Gamma Rays from Point Sources at Baksan Air Shower Array in 1984-1988: Proc. of Int. Workshop on Very High Energy Gamma Ray Astronomy (Crimea, USSR), 17-21 April, 1989. Ed. by A.A. Stepanyan, D.J. Fegan and M.F. Cawley, p. 187.

[9] M.V.S. Rao, K. Sivaprasad, B.V. Sreekantan and P.R. Vishwanath, First simultaneous detection of PeV energy burst from the Crab Nebula, Nature 1990; 347: 364-5

[10] V.V. Alexeenko, Yu.M. Andreyev, A.E. Chudakov, Ya.S. Elensky, A.S. Lidvansky, S.Kh. Ozrokov, Yu.V. Stenkin, V.A. Tizengauzen, L.J. Graham, J.L. Osborne and A.W. Wolfendale, The ultra-high energy gamma-ray burst from the Crab Nebula observed by the Baksan EAS array. 1992 J. Phys. G: Nucl. Part. Phys.; 18: L83-L88.

[11] M. Mayer et al., Rapid Camma-ray Flux Variability during the 2013 March Crab Nebula Flare. Astrophys. Journ. Lett. 2013; 775: L37. 